\title{
Quantifying Correlations Between Isovector Observables and the Density Dependence of Nuclear Symmetry Energy away from Saturation Density
}

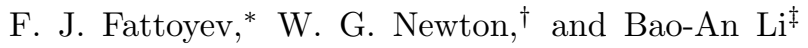 \\ Department of Physics and Astronomy, Texas A $6 M$ University-Commerce, Commerce, TX 75429, USA
}

(Dated: June 16, 2021)

\begin{abstract}
According to the Hugenholtz-Van Hove theorem, the nuclear symmetry energy $S(\rho)$ and its slope $L(\rho)$ at arbitrary densities can be decomposed in terms of the density and momentum dependence of the single-nucleon potentials in isospin-asymmetric nuclear matter which are potentially accessible to experiment. We quantify the correlations between several well-known isovector observables and $L(\rho)$ to locate the density range in which each isovector observable is most sensitive to the density dependence of the $S(\rho)$. We then study the correlation coefficients between those isovector observables and all the components of the $L(\rho)$. The neutron skin thickness of ${ }^{208} \mathrm{~Pb}$ is found to be strongly correlated with the $L(\rho)$ at a subsaturation density of $\rho=0.59 \rho_{0}$ through the density dependence of the first-order symmetry potential. Neutron star radii are found to be strongly correlated with the $L(\rho)$ over a wide range of supra-saturation densities mainly through both the density and momentum dependence of the first-order symmetry potential. Finally, we find that although the crust-core transition pressure has a complex correlation with the $L(\rho)$, it is strongly correlated with the momentum derivative of the first-order symmetry potential, and the density dependence of the second-order symmetry potential.
\end{abstract}

PACS numbers: 21.65.Cd, 21.65.Mn, 21.65.Ef, 26.60.Kp

Improving knowledge of the density dependence of the nuclear symmetry energy is an active endeavor due to its multifaceted impact in many areas of nuclear physics and astrophysics [1 [5], as well as in some issues regarding possible new physics beyond standard model 6 6 . Despite intensive efforts aimed at constraining the density dependence of the nuclear symmetry energy on both the experimental and theoretical fronts [11-13], its knowledge still remains largely uncertain even around nuclear saturation density $\rho_{0}$ [14]. Traditionally, the nuclear symmetry energy is expanded around $\rho_{0}$ as $S(\rho)=J+L \chi+$ $\frac{1}{2} K_{\text {sym }} \chi^{2}+\mathcal{O}\left(\chi^{3}\right)$ with $\chi \equiv\left(\rho-\rho_{0}\right) / 3 \rho_{0}$, and then individual parameters of this expansion-particularly $J$ and $L$ - are probed using experimental observables that are sensitive to their variation. In this way correlations between the density slope of the symmetry energy $L\left(\rho_{0}\right)$ and a multitude of isovector observables have been established, with neutron skins of heavy nuclei and radii of neutron stars 15 17. exhibiting notably strong correlations with $L$.

In this paper we begin examining the density dependence of these correlations, as well as the origin of the correlations by decomposing $L$ in terms of quantities that have a more direct physical meaning in finite nuclei and thus open up further potential experimental probes. By employing a least-squares covariance analysis with the correlation coefficient defined as

$$
C_{A B}=\frac{\operatorname{Cov}(A B)}{\operatorname{Var}(A) \operatorname{Var}(B)},
$$

we provide for the first time a proper statistical measure of correlations [18 20] between the density slope of the symmetry energy as a function of baryon density $L(\rho)$ and a selected number of isovector observables: (a) the neutron skin thickness, (b) radii of neutron stars and (c) the crust-core transition pressure. We will discuss the emergence of these correlations by decomposing the $L(\rho)$ in terms of the single-nucleon potentials in asymmetric nuclear matter as shown in Refs. 21 23. While in general the correlation coefficient $C_{A B}$ at a given density may not be able to assess systematic errors reflecting limitations of the model, the strongest correlation coefficient of almost +1 (or anticorrelation of almost -1 ) at a particular density should deliver a more universal modelindependent message. For this reason, we scan the correlation coefficients between the isovector observables and the $L(\rho)$ over a wide range of baryon densities.

Based on the Hugenholtz-Van Hove theorem [24, it was shown that both the nuclear symmetry energy $S(\rho)$ and its density slope $L(\rho)$ can be decomposed in terms of the single-nucleon potentials 23. For convenience, we will rewrite those expressions here as $S(\rho)=S_{1}(\rho)+$ $S_{2}(\rho)$ and $L(\rho)=L_{1}(\rho)+L_{2}(\rho)+L_{3}(\rho)+L_{4}(\rho)+L_{5}(\rho)$, where

$$
\begin{aligned}
& L_{1}(\rho)=\frac{2 \hbar^{2} k_{\mathrm{F}}^{2}}{6 m_{0}^{*}\left(\rho, k_{\mathrm{F}}\right)} \equiv 2 S_{1}(\rho) \\
& L_{2}(\rho)=-\left.\frac{\hbar^{2} k_{\mathrm{F}}^{3}}{6 m_{0}^{* 2}\left(\rho, k_{\mathrm{F}}\right)} \frac{\partial m_{0}^{*}(\rho, k)}{\partial k}\right|_{k=k_{\mathrm{F}}} \\
& L_{3}(\rho)=\frac{3}{2} U_{\mathrm{sym}, 1}\left(\rho, k_{\mathrm{F}}\right) \equiv 3 S_{2}(\rho) \\
& L_{4}(\rho)=\left.\frac{\partial U_{\mathrm{sym}, 1}(\rho, k)}{\partial k}\right|_{k=k_{\mathrm{F}}} \cdot k_{\mathrm{F}} \\
& L_{5}(\rho)=3 U_{\mathrm{sym}, 2}\left(\rho, k_{\mathrm{F}}\right) .
\end{aligned}
$$

The expressions above are valid at arbitrary baryon densities, where $m_{0}^{*}(\rho, k)$ is the nucleon effective mass in symmetric nuclear matter (SNM), while $U_{\text {sym }, 1}$ and 
$U_{\text {sym }, 2}$ are the first- and the second-order nuclear symmetry potentials defined as:

$$
\begin{aligned}
U_{\mathrm{sym}, \mathrm{i}}(\rho, k) & \left.\equiv \frac{1}{i} \frac{\partial^{i} U_{\mathrm{n}}(\rho, \alpha, k)}{\partial \alpha^{i}}\right|_{\alpha=0}= \\
& =\left.\frac{(-1)^{i}}{i} \frac{\partial^{i} U_{\mathrm{p}}(\rho, \alpha, k)}{\partial \alpha^{i}}\right|_{\alpha=0} .
\end{aligned}
$$

Here $U_{\mathrm{n}}$ and $U_{\mathrm{p}}$ are the single-neutron and single-proton potentials respectively, which generally depend on the baryion density $\rho$, the isospin asymmetry $\alpha$ and the amplitude of the nucleon momentum $k$. Physically, $S_{1}(\rho)$ and accordingly $L_{1}(\rho)$ represent the kinetic energy part of the symmetry energy that includes the isocalar effective mass contribution, $L_{2}(\rho)$ describes the momentum dependence of the nucleon effective mass, $S_{2}(\rho)$ hence $L_{3}(\rho)$ are due to the first-order symmetry potential contribution, $L_{4}(\rho)$ comes from the momentum dependence of the first-order symmetry potential, and $L_{5}(\rho)$ comes from the second-order symmetry potential. Since by definition, $L(\rho) \equiv 3 \rho \frac{\partial S(\rho)}{\partial \rho}$, it is then obvious that there is also a required closure relation between the density derivative of the first-order symmetry potential $U_{\mathrm{sym}, 1}$ and the magnitude of $U_{\mathrm{sym}, 2}$, in particular.

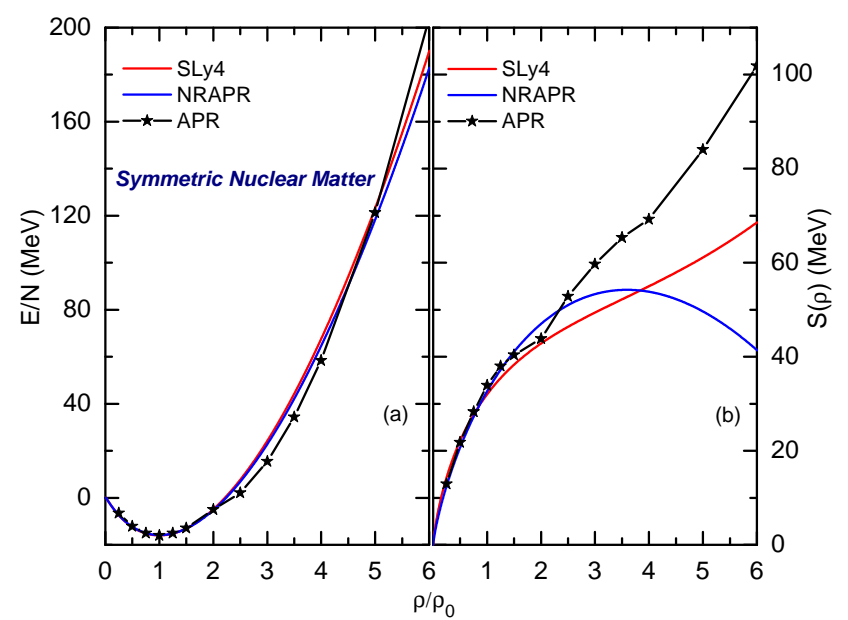

FIG. 1: (color online). Binding energy per nucleon in SNM (a) and the symmetry energy (b) as a function of baryon density $\rho / \rho_{0}$ for SLy 4 and NRAPR Skyrme EDFs.

The decomposition method considered above is quite general. In this exploratory study, we report our results for two Skyrme energy density functionals (EDFs) SLy4 25] and NRAPR 2] that have been widely used in the literature, both nuclear- and astrophysical. Since our aim is to study the isospin-dependent properties of asymmetric nuclear matter, SLy4 and NRAPR are natural choices for the following reasons. First, they predict an almost identical EOS of SNM (see the (a) panel of Fig.11). Therefore, the $L_{1}(\rho)$ term of the density slope is almost

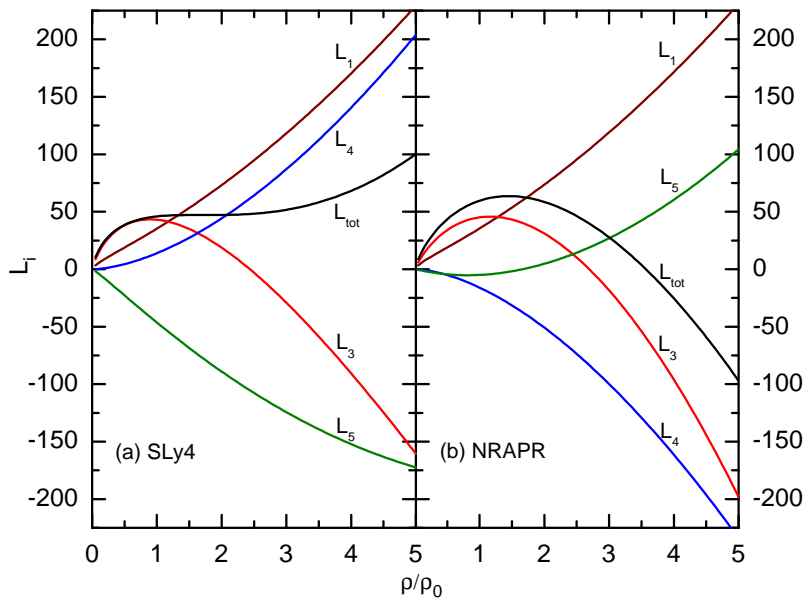

FIG. 2: (color online). Density dependence of the $L(\rho)$ and its decomposition for SLy4 and NRAPR Skyrme EDFs.

indistinguishable for these interactions due to the equivalence of the isoscalar effective mass, and the nuclear saturation density $\rho_{0}$ (see Fig. 2). Notice however that since the nucleon effective mass in Skyrme EDFs does not depend on momentum, the $L_{2}(\rho)$ term becomes identically zero. Second, both Sly4 and NRAPR reproduce the symmetry energy predicted by the APR EOS [26] up to $\sim 1.5 \rho_{0}$ (see the (b) panel of Fig. 1). Whereas a similar density dependence of $L_{3}(\rho)$-hence $S_{2}(\rho)$ or $U_{\text {sym }, 1}$ - is observed in these interactions, both $L_{4}(\rho)$ and $L_{5}(\rho)$ terms have a completely different density dependence (see Fig. 2). Consequently these models have a totally different density dependence of the symmetry energy at supra-saturation densities of $\rho \gtrsim 1.5 \rho_{0}$.

As a starting point, using the currently accepted uncertainty ranges by the community we fix the isoscalar properties of bulk nuclear matter such as the nuclear saturation density $\rho_{0}$, the binding energies per nucleon in SNM at saturation $B\left(\rho_{0}\right)$ and at twice saturation density $B\left(2 \rho_{0}\right)$, the incompressibility coefficient of nuclear matter $K_{0}$, the nucleon isoscalar effective mass $m_{\mathrm{s}}^{*}\left(\rho_{0}\right)$, and the macroscopic gradient coefficient $G_{\mathrm{s}}[25]$ at a $2 \%$ level, while allow the isovector effective mass $m_{\mathrm{v}}^{*}\left(\rho_{0}\right)$, and the symmetry-gradient coefficient $G_{\mathrm{v}}$ to have a $20 \%$ theoretical error-bars [27]. We do not a priory assume any error-bars on the symmetry energy parameters, hence they have not been included in the $\chi^{2}$. Rather we include a conservative range of theoretical data points for the neutron-matter energy at densities $0.04<\rho / \rho_{0}<0.12 \mathrm{fm}^{-3}$ that were calculated using quantum Monte Carlo calculations with chiral effective field theory interactions [28].

In Fig. 3 we display the correlation coefficients between the $L(\rho)$ as a function of the baryon density and the neutron skin thicknesses of ${ }^{208} \mathrm{~Pb}$ and ${ }^{48} \mathrm{Ca}$. An updated 


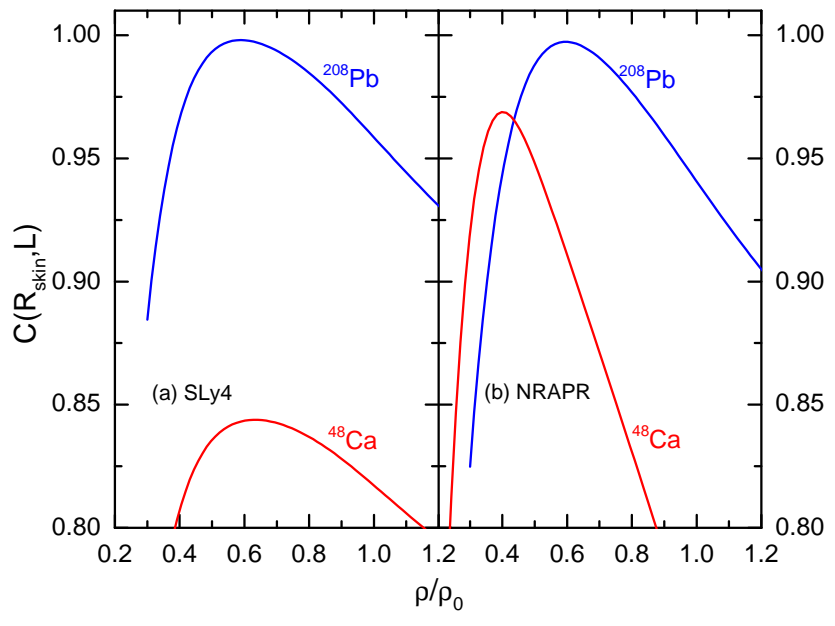

FIG. 3: (color online). Correlation coefficients between the $L(\rho)$ and the neutron skin thicknesses of ${ }^{208} \mathrm{~Pb}$ and ${ }^{48} \mathrm{Ca}$ calculated using SLy4 (left) and NRAPR (right) Skyrme EDFs.

measurement of the skin thickness in ${ }^{208} \mathrm{~Pb}$, and a proposal for measuring the skin thickness in ${ }^{48} \mathrm{Ca}$ has been recently approved at the Thomas Jefferson National Accelerator Facility [29. As evident from the figure, although the strong correlation between $R_{\text {skin }}\left({ }^{208} \mathrm{~Pb}\right)$ and the slope of the symmetry energy at saturation $L\left(\rho_{0}\right)$ is present consistent with previous studies, the strongest correlation coefficient of almost +1 appears only at a much lower density of about $\rho / \rho_{0}=0.59$ for both EDFs. This means that a measurement of the neutron skin in ${ }^{208} \mathrm{~Pb}$ would uniquely determine the slope of the symmetry energy at this particular sub-saturation density. Indeed, a recent systematic study also showed that a strong correlation coefficient between $R_{\text {skin }}\left({ }^{208} \mathrm{~Pb}\right)$ and $L(\rho)$ emerges at a sub-saturation cross density of $\rho_{\mathrm{c}} \approx 0.11$ $\mathrm{fm}^{-3}[30$. This result should not come as a surprise, since only about one-third of the nucleons in ${ }^{208} \mathrm{~Pb}$ occupy the saturation density area, which therefore explains why the neutron skin thickness should constrain the $L(\rho)$ not at $\rho_{0}$, but at a characteristic density in finite nuclei, which is localized close to a mean value of the density of nuclei [31. One should note that the neutron skin is formed as a result of the competition between the surface tension and the pressure of neutrons in heavy nuclei, the latter being closely related to the $L(\rho)$. Hence the greater is the $L(\rho)$ the thicker is the skin [15]17. This simple picture is of course relevant for heavy nuclei, where the mean-field approximation is adequate. In the case of light nuclei such as ${ }^{48} \mathrm{Ca}$ the mean-field approximation may not be sufficient, and one should expect beyond mean-field effects to crop up. Indeed, as seen in Fig. 3 although the correlation remains as strong at a sub-saturation density, it is not close to +1 , leaving a very rich unexplored physics behind. Moreover, the strongest correlation co-

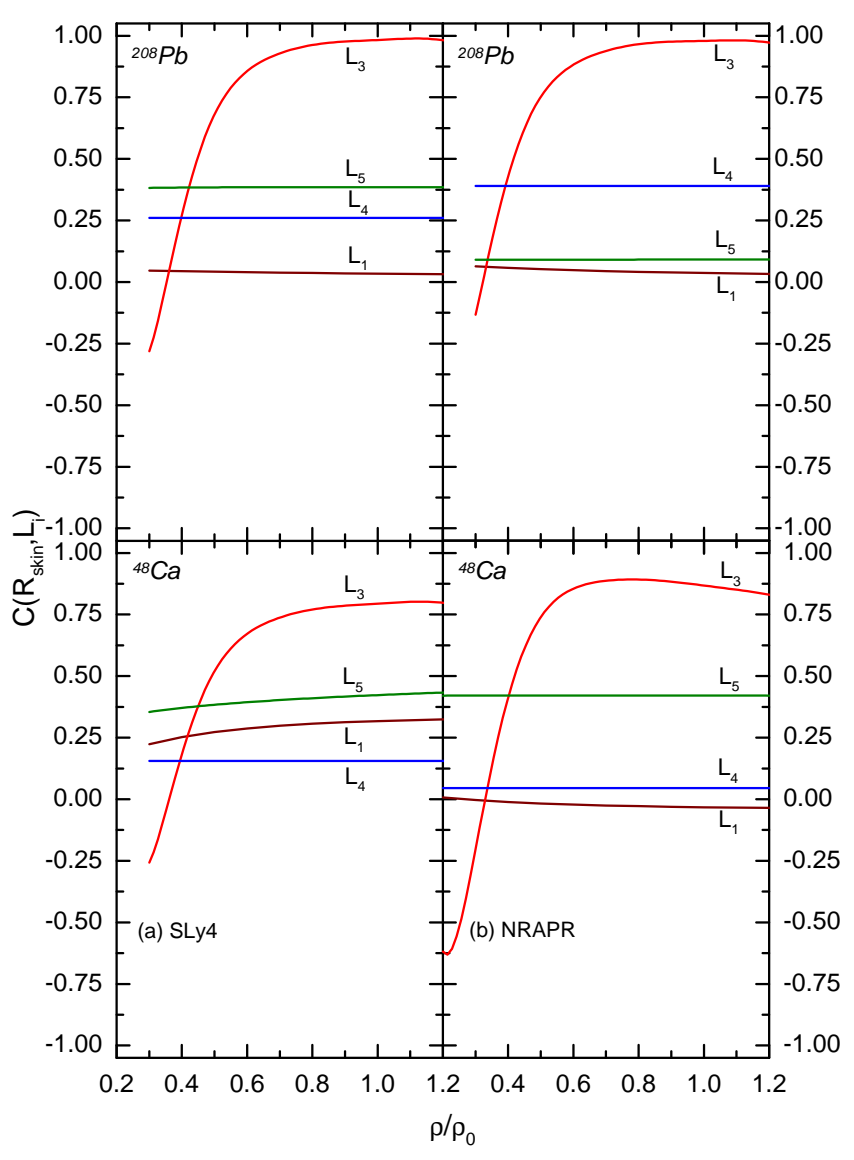

FIG. 4: (color online). Correlation coefficients between various terms in the $L(\rho)$ and the neutron skin thicknesses of ${ }^{208} \mathrm{~Pb}$ (upper) and ${ }^{48} \mathrm{Ca}$ (lower) calculated using SLy4 (left) and NRAPR (right) Skyrme EDFs.

efficients occur at different densities of $\rho=0.63 \rho_{0}$ for SLy 4 and at $\rho=0.39 \rho_{0}$ for NRAPR. Thus, simultaneous measurement of $R_{\text {skin }}$ in both ${ }^{208} \mathrm{~Pb}$ and ${ }^{48} \mathrm{Ca}$, are very important as they do not only help map out the density dependence of the symmetry energy in a broader subsaturation density region, but should also provide complementary information on the structure of neutron-rich calcium isotope ${ }^{48} \mathrm{Ca}[29$. Next, in Fig. 4 we display correlation coefficients between $R_{\text {skin }}$ and the individual decomposed terms of $L(\rho)$ at various sub-saturation densities. Except $L_{3}(\rho)$, or $U_{\text {sym }, 1}$, all other terms show almost no sign of a correlation. In fact, one should not expect any correlation with $L_{1}(\rho)$ term as it is purely isocalar in nature. The little correlation with $L_{4}(\rho)$ and $L_{5}(\rho)$ indicate that the size of the neutron skin is not particularly sensitive to the second-order symmetry potential and the momentum dependence of the first order symmetry potential.

Since the pressure of neutron-rich matter also supports neutron stars against gravitational collapse albeit at different densities than found at the center of finite nuclei, 


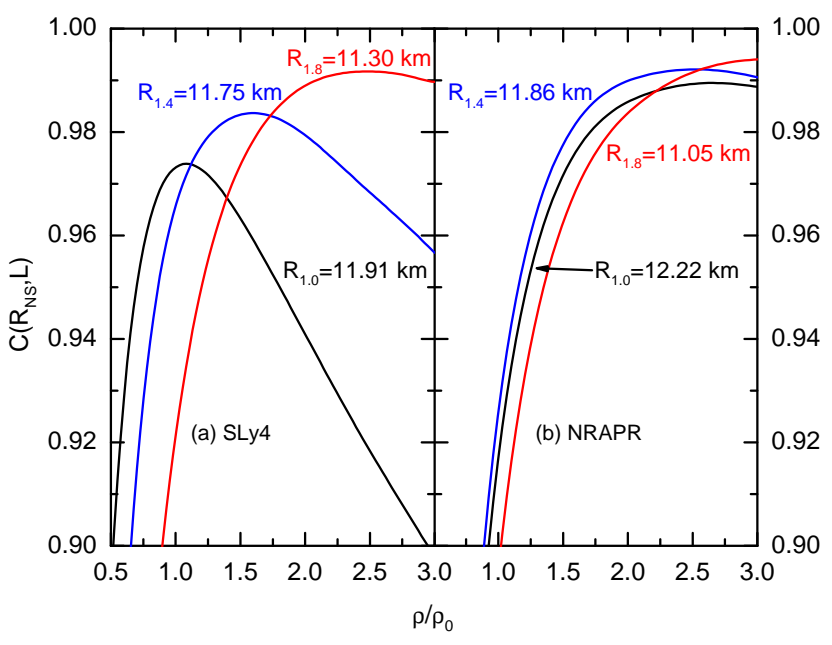

FIG. 5: (color online). Correlation coefficients between $L(\rho)$ and the 1.0-, 1.4-, and 1.8-solar mass neutron star radii as a function of the baryon density calculated using SLy4 (left) and NRAPR (right) Skyrme EDFs.

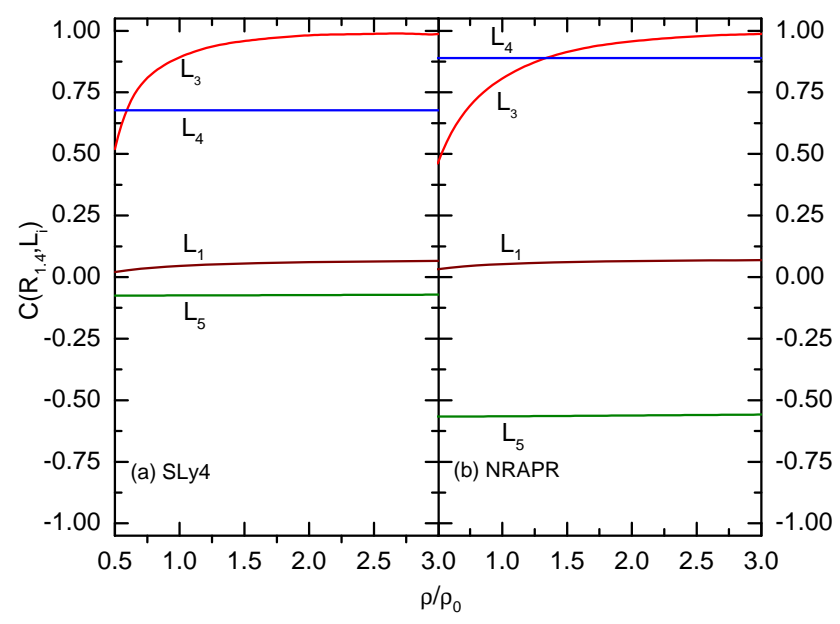

FIG. 6: (color online). Correlation coefficients between various terms in the $L(\rho)$ as a function of the baryon density and a canonical 1.4-solar mass neutron star radii are calculated using SLy4 (left) and NRAPR (right) Skyrme EDFs.

one should also expect the strong correlation to emerge between the $L(\rho)$ and the neutron star radii. This correlation is complicated by the fact that the radius of a neutron star samples the symmetry energy over a range from half saturation density up to several times saturation density; one should expect potentially significant variations in the correlation coefficient as a function of density from EOS to EOS. We demonstrate this by displaying the correlation coefficients between the neutron star radii and the $L(\rho)$ as a function of density for both SLy4 and NRAPR models in Fig. 5. In the case of SLy4, the radius of a 1.0-solar mass neutron star shows a strong correlation with the density slope at saturation. As the mass of the neutron star increases, the strongest correlation shifts to the $L(\rho)$ at higher densities, e.g. at $1.5 \rho_{0}$ for a 1.4-solar mass neutron star, and at $2.5 \rho_{0}$ for a 1.8 -solar mass neutron star. Moreover, the correlation coefficient remains almost flat for higher densities in a 1.8-solar mass neutron star. Higher mass stars sample the internal pressure at a higher average density. SLy4 has monotonically increasing $L(\rho)$, and thus the internal pressure at higher densities will continue to be dominated by the symmetry energy contribution.

NRAPR exhibits a different evolution of the correlation coefficient with neutron star mass, with a much less pronounced increase in its peak as we move to higher masses. The correlation for 1.0 and 1.4-solar mass stars peak at a densities of around $\sim 2.5 \rho_{0}$ while the correlation for a 1.8-solar mass star peaks at the slightly higher density of $\sim 3.5 \rho_{0} . \quad L(\rho)$ at supra-saturation densities for NRAPR is non-monotonic, peaking at around $1.5 \rho_{0}$ before falling to zero at $3.5 \rho_{0}$ - the density range within which the radius shows its peak correlation with $L$. Beyond the peak $L(\rho)$, the contributions to the internal pressure from the higher-order symmetry coefficients and from the symmetric part of the EOS will become steadily more important, thus quenching the correlation with $L$. The two behaviors of $L(\rho)$ exhibited by the two Skyrme models here broadly bracket the types of behaviors seen in all Skyrme models, and the behaviors of the correlation coefficient between radius and $L(\rho)$ with increasing NS mass can be expected to similarly bracket the range of possible behaviors in such models. Nevertheless, for both models the strongest correlation coefficient for a 1.4-solar mass star emerges with $L(\rho)$ at supra-saturation densities, between $1.5 \rho_{0}$ and $2 \rho_{0}$. The need for the symmetry energy in this range for the determination of neutron star radii was also empirically observed in Ref. [4]. Thus measurements of the neutron skin in finite nuclei and the radius of neutron stars probe quite different density regimes, unsurprisingly. The latter of these regimes may be tested with collisions of very neutron-rich heavy ions at different beam energies 32 .

We show the correlation coefficients between the radius of a $1.4 M_{\odot}$ star and the components of $L(\rho)$ in Fig. 6. Similar to the neutron skin case, no correlation is found with $L_{1}(\rho)$, and relatively mild correlations or anti-correlations are found with $L_{4}(\rho)$ and $L_{5}(\rho)$. It is again the $L_{3}(\rho)$ term that appears to have strongest correlation at higher densities. Recall that $L_{3}(\rho)=\frac{3}{2} U_{\mathrm{sym}, 1}$. This indicates that refinements in extracting $U_{\text {sym }, 1}$ from the measurement of the nucleon optical model potentials, and from heavy ion collision observables, would improve our predictions of neutron skins and neutron star radii.

Finally, we discuss our results for the crust-core transition pressure, the most important correlate with the thickness, mass, and moment of inertia of neutron star's crust [33, 34. Several methods have been used to de- 


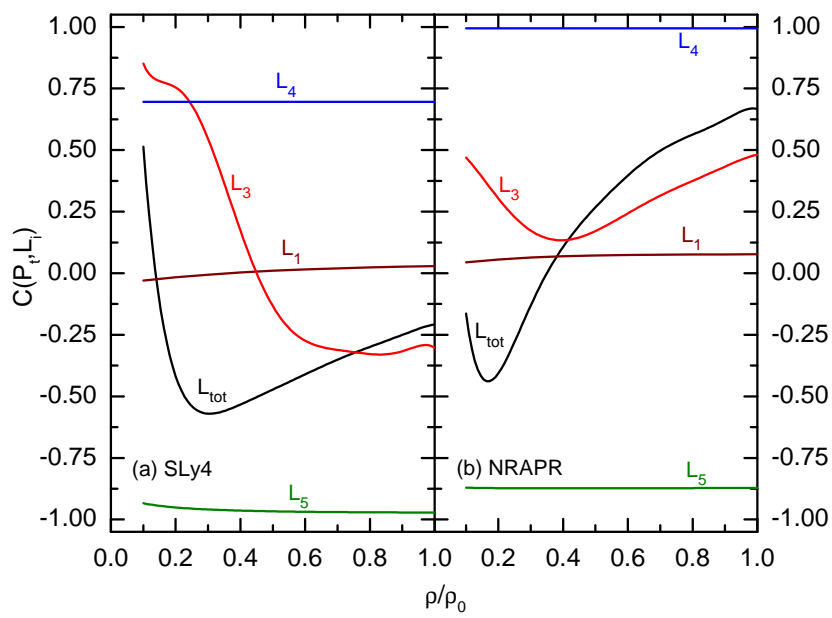

FIG. 7: (color online). Correlation coefficients between various terms in the $L(\rho)$ and the crust-core transition pressure are calculated using SLy4 (left) and NRAPR (right) models.

termine the crust-core transition properties [16, 35] 37]. Unlike the neutron skin thickness and the radii of neutron stars, the crust-core transition pressure cannot be determined entirely by the pressure of pure-neutron rich matter itself. Indeed, in the simplest case of the thermodynamical approach the following mechanical stability condition, $\left(\frac{\partial P}{\partial \rho}\right)_{\mu}>0$, must be satisfied in order for the system to be stable against small density fluctuations, where $\mu$ is the chemical potential. Since the density derivative of the pressure is quite complicated, a complex correlation between the transition pressure and the $L(\rho)[33,35,37,38$, must therefore emerge. We determine the transition pressure from a compressible liquid drop model of the crust outlined in Ref. [39]. By plotting the correlation coefficients between various terms in the $L(\rho)$ and the crust-core transition pressure in Fig. 7 we observe that the values of $L_{4}(\rho)$ and $L_{5}(\rho)$-that is the momentum derivative of $U_{\mathrm{sym}, 1}$ and the magnitude of $U_{\mathrm{sym}, 2}$ - are most important in the determination of the transition pressure. The complex correlation between the crust-core transition pressure and the $L(\rho)$ appears to originate from the complicated behavior of the correlation with $L_{3}(\rho)$ coupled with the balance between the strong correlation between the crust-core transition pressure and $L_{4}(\rho)$, and the similarly strong anti-correlation with $L_{5}(\rho)$, which together complicate the emergence of correlation with the total density slope. Thus extracting the density and momentum dependence of $U_{\mathrm{sym}, 1}$, and the value of $U_{\mathrm{sym}, 2}$ are both very crucial in determining the crust-core transition pressure, which plays critical role in understanding many phenomena related to the neutron star crust [40].

In summary, we have quantitatively mapped the correlations between $L(\rho)$ and the neutron skin thickness, radii of neutron stars and the crust-core transition pressure over a wide range of densities for two widely used Skyrme models SLy4 and NRAPR which have similar symmetric nuclear matter EOSs but diverging behaviors of $L(\rho)$ at supra-saturation densities. We have also calculated the correlation coefficients between the symmetry potential component of $L$ at saturation density and the same set of isovector observables. We found that for the neutron skin thickness of ${ }^{208} \mathrm{~Pb}$ the strongest correlation appears at a subsaturation density of $\rho=0.59 \rho_{0}$, and that the origin of this correlation is found to be tied to the magnitude of the first-order symmetry potential $U_{\text {sym }, 1}$. A similarly strong correlation exists with the radius of neutron stars and $L(\rho)$ over a wide range of supra-saturation densities. The behavior of the correlation is seen to depend on the behavior of $L(\rho)$ at supra-saturation densities. If $L(\rho)$ continues to monotonically increase, then the peak correlation of radius with $L$ occurs at higher densities for higher mass stars. If $L(\rho)$ increases to a maximum and starts decreasing above a certain supra-saturation density, then the peak correlation of radius with $L$ occurs within a similar density range independent of mass. The radius is also found to correlate most strongly with the magnitude of the first-order symmetry potential $U_{\text {sym }, 1}$.

The crust-core transition pressure, on the other hand, is found to be strongly correlated not with the value of $U_{\text {sym }, 1}$ but with its momentum derivative term and the magnitude of $U_{\mathrm{sym}, 2}$. Future improvements in extracting the density and momentum dependence of first- and second-order symmetry potentials from optical model analysis of nucleon-nucleus scattering and at facilities for rare isotope beams will therefore provide strong constraints on the density dependence of the symmetry energy.

We would like to thank Prof. Lie-Wen Chen and Prof. Jorge Piekarewicz for fruitful discussions. This work was supported in part by the National Aeronautics and Space Administration under Grant No. NNX11AC41G issued through the Science Mission Directorate, and the National Science Foundation under Grant No. PHY1068022 .

* Electronic address: farrooh.fattoyev@tamuc.edu

† Electronic address: william.newton@tamuc.edu

¥ Electronic address: bao-an.li@tamuc.edu

[1] B.-A. Li, A. Ramos, G. Verde, and I. Vidana, eds., "Topical Issue on Nuclear Symmetry Energy", Eur. Phys. J. A50, No. 2 (2014).

[2] A. W. Steiner, M. Prakash, J. M. Lattimer, and P. J. Ellis, Phys. Rept. 411, 325 (2005), nucl-th/0410066.

[3] V. Baran, M. Colonna, V. Greco, and M. Di Toro, Phys. Rept. 410, 335 (2005), nucl-th/0412060.

[4] J. M. Lattimer and M. Prakash, Phys. Rept. 442, 109 (2007), astro-ph/0612440.

[5] B.-A. Li, L.-W. Chen, and C. M. Ko, Phys. Rept. 464, 
113 (2008), 0804.3580.

[6] C. J. Horowitz, S. J. Pollock, P. A. Souder, and R. Michaels, Phys. Rev. C63, 025501 (2001), nuclth/9912038.

[7] T. Sil, M. Centelles, X. Vinas, and J. Piekarewicz, Phys. Rev. C71, 045502 (2005), nucl-th/0501014.

[8] P. G. Krastev and B.-A. Li, Phys.Rev. C76, 055804 (2007), nucl-th/0702080.

[9] D.-H. Wen, B.-A. Li, and L.-W. Chen, Phys. Rev. Lett. 103, 211102 (2009), 0908.1922.

[10] W. Lin, B.-A. Li, L.-W. Chen, D.-H. Wen, and J. Xu (2013), 1311.3216.

[11] J. M. Lattimer and Y. Lim, Astrophys.J. 771, 51 (2013), 1203.4286 .

[12] J. M. Lattimer, Ann. Rev. Nucl. Part. Sci. 62, 485 (2012), 1305.3510.

[13] B.-A. Li and X. Han, Phys. Lett. B727, 276 (2013), 1304.3368.

[14] F. J. Fattoyev and J. Piekarewicz, Phys. Rev. Lett. 111, 162501 (2013), 1306.6034.

[15] B. A. Brown, Phys. Rev. Lett. 85, 5296 (2000).

[16] C. J. Horowitz and J. Piekarewicz, Phys. Rev. Lett. 86, 5647 (2001), astro-ph/0010227.

[17] R. J. Furnstahl, Nucl. Phys. A706, 85 (2002), nuclth/0112085.

[18] P.-G. Reinhard and W. Nazarewicz, Phys. Rev. C81, 051303 (2010), 1002.4140.

[19] F. J. Fattoyev and J. Piekarewicz, Phys. Rev. C84, 064302 (2011), 1109.1576.

[20] F. J. Fattoyev and J. Piekarewicz, Phys. Rev. C86, 015802 (2012), 1203.4006.

[21] C. Xu, B.-A. Li, and L.-W. Chen, Phys. Rev. C82, 054607 (2010), 1006.4321.

[22] C. Xu, B.-A. Li, L.-W. Chen, and C. M. Ko, Nucl. Phys. A865, 1 (2011), 1004.4403.

[23] R. Chen, B.-J. Cai, L.-W. Chen, B.-A. Li, X.-H. Li, et al., Phys. Rev. C85, 024305 (2012), 1112.2936.

[24] N. Hugenholtz and L. van Hove, Physica 24, 363 (1958).

[25] E. Chabanat, J. Meyer, P. Bonche, R. Schaeffer, and P. Haensel, Nucl. Phys. A627, 710 (1997).

[26] A. Akmal, V. R. Pandharipande, and D. G. Ravenhall, Phys. Rev. C58, 1804 (1998), nucl-th/9804027.

[27] L.-W. Chen, C. M. Ko, B.-A. Li, and J. Xu, Phys. Rev. C82, 024321 (2010), 1004.4672.

[28] A. Gezerlis, I. Tews, E. Epelbaum, S. Gandolfi, K. Hebeler, et al., Phys. Rev. Lett. 111, 032501 (2013), 1303.6243.

[29] C. Horowitz, K. Kumar, and R. Michaels, Eur.Phys.J. A50, 48 (2014), 1307.3572.

[30] Z. Zhang and L.-W. Chen, Phys. Lett. B726, 234 (2013), 1302.5327.

[31] E. Khan, J. Margueron, and I. Vidana, Phys. Rev. Lett. 109, 092501 (2012), 1204.0399.

[32] C. Horowitz, E. Brown, Y. Kim, W. Lynch, R. Michaels, A. Ono, J. Piekarewicz, M. B. Tsang, and H. H. Walter, (2014), 1401.5839.

[33] F. J. Fattoyev and J. Piekarewicz, Phys. Rev. C82, 025810 (2010), 1006.3758.

[34] J. Piekarewicz, F. J. Fattoyev, and C. J. Horowitz (2014), 1404.2660

[35] J. Xu, L.-W. Chen, B.-A. Li, and H.-R. Ma, Astrophys. J. 697, 1549 (2009), 0901.2309.

[36] S. S. Avancini, S. Chiacchiera, D. P. Menezes, and C. Providencia, Phys. Rev. C82, 055807 (2010),
1010.3644.

[37] C. Ducoin, J. Margueron, C. Providencia, and I. Vidana, Phys. Rev. C83, 045810 (2011), 1102.1283.

[38] B.-A. Li and C. Ko, Nucl. Phys. A618, 498 (1997), nuclth/9701049.

[39] W. G. Newton, M. Gearheart, and B.-A. Li, Astrophys. J. Suppl. 204, 9 (2013), 1110.4043.

[40] W. G. Newton, J. Hooker, M. Gearheart, K. Murphy, D.-H. Wen, F. J. Fattoyev, and B.-A. Li, Eur. Phys. J. A50, 41 (2014). 